\title{
Characterization of Globally Lipschitz Nemytskiu Operators Between Spaces of Set-Valued Functions of Bounded $\varphi$-Variation in the Sense of Riesz
}

by

\author{
N. MERENTES and J. L. SÁNCHEZ HERNÁNDEZ
}

Presented by Stanistaw KWAPIEN

\begin{abstract}
Summary. Let $(X,\|\cdot\|)$ and $(Y,\|\cdot\|)$ be two normed spaces and $K$ be a convex cone in $X$. Let $C C(Y)$ be the family of all non-empty convex compact subsets of $Y$. We consider the Nemytskii operators, i.e. the composition operators defined by $(N u)(t)=H(t, u(t))$, where $H$ is a given set-valued function. It is shown that if the operator $N$ maps the space $R V_{\varphi_{1}}([a, b] ; K)$ into $R W_{\varphi_{2}}([a, b] ; C C(Y)$ ) (both are spaces of functions of bounded $\varphi$-variation in the sense of Riesz), and if it is globally Lipschitz, then it has to be of the form $H(t, u(t))=A(t) u(t)+B(t)$, where $A(t)$ is a linear continuous set-valued function and $B$ is a set-valued function of bounded $\varphi_{2}$-variation in the sense of Riesz. This generalizes results of G. Zawadzka [12], A. Smajdor and W. Smajdor [11], N. Merentes and K. Nikodem [5], and N. Merentes and S. Rivas [7].
\end{abstract}

1. Introduction. In [11] A. Smajdor and W. Smajdor proved that every globally Lipschitz Nemytskiı operator $(N u)(t)=H(t, u(t))$ mapping the space $\operatorname{Lip}([a, b] ; C C(Y))$ into itself admits the following representation:

$$
(N u)(t)=A(t) u(t)+B(t), \quad u \in \operatorname{Lip}([a, b] ; C C(Y)), t \in[a, b],
$$

where $A(t)$ is a linear continuous set-valued function and $B$ is a set-valued function belonging to the $\operatorname{space} \operatorname{Lip}([a, b] ; C C(Y))$. The first such theorem for single-valued functions was proved by J. Matkowski [3] in the space of Lipschitz functions. A similar characterization of the Nemytskil operator has also been obtained by G. Zawadzka [12] in the space of set-valued functions of bounded variation in the classical Jordan sense. For single-valued functions

2000 Mathematics Subject Classification: Primary 47B33; Secondary 26E25.

Key words and phrases: Nemytskiu operator, set-valued functions, Jensen equation, composition operator, $\varphi$-variation in the sense of Riesz.

This research was partially supported by CDCH-CONICIT. 
it was proved by J. Matkowski and J. Miś [4]. N. Merentes and K. Nikodem [5] and N. Merentes and S. Rivas [7] proved an analogous theorem in the space of set-valued functions of bounded $p$-variation in the sense of Riesz. Also, they proved a similar result in the case that the Nemytskil operator $N$ maps the space of functions of bounded $p$-variation in the sense of Riesz into the space of set-valued functions of bounded $q$-variation in the sense of Riesz, where $1 \leq q \leq p<\infty$, and $N$ is globally Lipschitz.

The aim of this paper is to prove an analogous result in the case that the Nemytskiı operator $N$ maps the space $R V_{\varphi_{1}}([a, b] ; K)$ of set-valued functions of bounded $\varphi_{1}$-variation in the sense of Riesz into the space $R W_{\varphi_{2}}([a, b] ; C C(Y))$ of set-valued functions of bounded $\varphi_{2}$-variation in the sense of Riesz and $N$ is globally Lipschitz.

2. Preliminary results. In this section we introduce some definitions and recall known results concerning the Riesz $\varphi$-variation.

Definition 2.1. By a $\varphi$-function we mean a non-decreasing continuous function $\varphi:[0, \infty] \rightarrow[0, \infty]$ such that $\varphi(x)=0$ if and only if $x=0$, and $\varphi(x) \rightarrow \infty$ as $x \rightarrow \infty$.

Definition 2.2. Let $(X,\|\cdot\|)$ be a normed space and $\varphi$ be a $\varphi$-function. Given a function $u:[a, b] \rightarrow X$ and a partition $\pi: a=t_{0}<\cdots<t_{n}=b$ of the interval $[a, b]$, we define

$$
\sigma_{\varphi}(u ; \pi):=\sum_{i=1}^{n} \varphi\left(\frac{\left\|u\left(t_{i}\right)-u\left(t_{i-1}\right)\right\|}{\left|t_{i}-t_{i-1}\right|}\right)\left|t_{i}-t_{i-1}\right| .
$$

Denote by $\Pi$ the set of all partitions $\pi$ of $[a, b]$. Then the number

$$
V_{\varphi}(u)=V_{\varphi}(u,[a, b] ; X):=\sup \left\{\sigma_{\varphi}(u ; \pi): \pi \in \Pi\right\},
$$

is called the Riesz $\varphi$-variation $u$ on $[a, b]$. The function $u$ is said to be of bounded $\varphi$-variation if $V_{\varphi}(u)<\infty$.

Denote by $R V_{\varphi}([a, b])$ the set of all functions $u:[a, b] \rightarrow X$ such that $V_{\varphi}(\lambda u)<\infty$ for some $\lambda>0$. If $\varphi$ is convex, then $R V_{\varphi}([a, b])$ is a Banach space endowed with the norm

$$
\|u\|_{\varphi}:=\|u(a)\|+\inf \left\{\varepsilon>0: V_{\varphi}(u / \varepsilon) \leq 1\right\} .
$$

Also, if $u(a)=0$ we set

$$
\|u\|_{\varphi, 0}=\inf \left\{\varepsilon>0: V_{\varphi}(u / \varepsilon) \leq 1\right\} .
$$

It is known (see [1] or [2]) that convex $\varphi$-function $\varphi$ with $\lim _{t \rightarrow \infty} \varphi(t) / t$ $=r<\infty$ the following inequality holds:

$$
\|u\|_{\varphi, 0} \leq r V_{1}(u) \leq r\left(\varphi^{-1}(1)+(b-a)\right)\|u\|_{\varphi, 0} \quad \text { for all } u \in R V_{\varphi}[a, b],
$$


where $V_{1}(u):=\sup _{\pi \in \Pi} \sum_{i=1}^{n}\left\|u\left(t_{i}\right)-u\left(t_{i-1}\right)\right\|$. Consequently, $R V_{\varphi}[a, b]=$ $B V[a, b]$, and there exist constants $K_{1}$ and $K_{2}$ such that

$$
\|u\|_{\varphi} \leq K_{1}\|u\|_{B V[a, b]} \leq K_{2}\|u\|_{\varphi} \quad \text { for all } u \in R V_{\varphi}[a, b] .
$$

We need the following definition

DeFinition 2.3. Let $\varphi$ be a $\varphi$-function. We say that $\varphi$ satisfies condition $\infty_{1}$ if

$$
\limsup _{t \rightarrow \infty} \frac{\varphi(t)}{t}=\infty
$$

For $\varphi$ convex, (2.6) is just $\lim _{t \rightarrow \infty} \varphi(t) / t=\infty$.

Let $C C(X)$ be the family of all non-empty convex compact subsets of $X$, and let $D$ be the Pompeiu-Hausdorff metric in $C C(X)$, i.e.

$$
D(A, B):=\inf \{t>0: A \subseteq B+t S, B \subseteq A+t S\},
$$

where $S=\{y \in X:\|y\| \leq 1\}$, or equivalently,

$$
D(A, B)=\max \{e(A, B), e(B, A): A, B \in C C(X)\},
$$

where

$$
e(A, B)=\sup \{d(x, B): x \in A\}, \quad d(x, B)=\inf \{d(x, y): y \in B\} .
$$

Definition 2.4. Let $\varphi$ be a $\varphi$-function and $F:[a, b] \rightarrow C C(X)$. We say that $F$ has bounded $\varphi$-variation in the Riesz sense if

$$
W_{\varphi}^{R}(F ;[a, b]):=\sup _{\pi \in \Pi} \sum_{i=1}^{n} \varphi\left(\frac{D\left(F\left(t_{i}\right), F\left(t_{i-1}\right)\right)}{\left|t_{i}-t_{i-1}\right|}\right)\left|t_{i}-t_{i-1}\right|<\infty .
$$

Set

$$
R W_{\varphi}^{*}[a, b]:=\left\{F:[a, b] \rightarrow C C(X): W_{\varphi}^{R}(F ;[a, b])<\infty\right\},
$$

(2.10) $R W_{\varphi}[a, b]:=\left\{F:[a, b] \rightarrow C C(X): W_{\varphi}^{R}(\lambda F)<\infty\right.$ for some $\left.\lambda>0\right\}$, both equipped with the metric

$$
D_{\varphi}\left(F_{1}, F_{2}\right):=D\left(F_{1}(a), F_{2}(a)\right)+\inf \left\{\varepsilon>0: W_{\varphi}\left(F_{1} / \varepsilon, F_{2} / \varepsilon\right) \leq 1\right\},
$$

where

$$
W_{\varphi}\left(F_{1}, F_{2}\right):=\sup _{\pi \in \Pi} \sum_{i=1}^{n} \varphi\left(\frac{D\left(F_{1}\left(t_{i}\right)+F_{2}\left(t_{i-1}\right), F_{1}\left(t_{i-1}\right)+F_{2}\left(t_{i}\right)\right)}{\left|t_{i}-t_{i-1}\right|}\right)\left|t_{i}-t_{i-1}\right| .
$$

Now, let $(X,\|\cdot\|),(Y,\|\cdot\|)$ be two normed spaces and $K$ be a convex cone in $X$. Given a set-valued function $H:[a, b] \times K \rightarrow C C(Y)$ we consider the Nemytskic operator $N: K^{[a, b]} \rightarrow Y^{[a, b]}$ generated by $H$, i.e.

$$
(N u)(t):=H(t, u(t)), \quad u \in K^{[a, b]}, \quad t \in[a, b] .
$$

We denote by $L(K ; C C(Y))$ the space of all set-valued linear functions $A$ : $K \rightarrow C C(Y)$, i.e. additive and positively homogeneous. 
In the proof of the main results of this paper we will use some facts which we list here as lemmas.

Lemma 2.1 (see [9, Lemma 3]). Let $(X,\|\|$.$) be a normed space and let$ $A, B, C$ be subsets of $X$. If $A, B$ are convex and $C$ is non-empty and bounded, then

$$
D(A+C, B+C)=D(A, B) .
$$

Lemma 2.2 (see $[8$, Th. 5.6]). Let $(X,\|\cdot\|),(Y,\|\cdot\|)$ be normed spaces and $K$ be a convex cone in $X$. A set-valued function $F:[a, b] \rightarrow C C(Y)$ satisfies the Jensen equation

$$
F\left(\frac{x+y}{2}\right)=\frac{1}{2}(F(x)+F(y)), \quad x, y \in K,
$$

if and only if there exists an additive set-valued function $A: K \rightarrow C C(Y)$ and $a$ set $B \in C C(Y)$ such that $F(x)=A(x)+B, x \in K$.

We will extend the result of N. Merentes and K. Nikodem [5] to set-valued functions of $\varphi$-bounded variation.

\section{Main results}

LEMMA 3.1. If $\varphi$ is a convex $\varphi$-function that satisfies condition $\infty_{1}$ and $F \in R W_{\varphi}[a, b]$, then $F:[a, b] \rightarrow C C(X)$ is continuous.

Proof. Since $F \in R W_{\varphi}[a, b]$, there exists $M>0$ such that

$$
\sum_{i=1}^{n} \varphi\left(\frac{D\left(F\left(t_{i}\right), F\left(t_{i-1}\right)\right)}{\left|t_{i}-t_{i-1}\right|}\right)\left|t_{i}-t_{i-1}\right| \leq M
$$

for all partitions of $[a, b]$; in particular given $t, t_{0} \in[a, b]$, we have

$$
\varphi\left(\frac{D\left(F(t), F\left(t_{0}\right)\right)}{\left|t-t_{0}\right|}\right)\left|t-t_{0}\right| \leq M
$$

Since $\varphi$ is a convex $\varphi$-function, from the last inequality we get

$$
D\left(F(t), F\left(t_{0}\right)\right) \leq \frac{\varphi^{-1}\left(\frac{M}{\left|t-t_{0}\right|}\right)}{\frac{1}{\left|t-t_{0}\right|}} .
$$

By $(2.6)$,

$$
\lim _{t \rightarrow t_{0}} D\left(F(t), F\left(t_{0}\right)\right) \leq \lim _{t \rightarrow t_{0}} \frac{\varphi^{-1}\left(\frac{M}{\left|t-t_{0}\right|}\right)}{\frac{1}{\left|t-t_{0}\right|}}=\lim _{t \rightarrow \infty} \frac{M t}{\varphi(t)}=0 .
$$

This proves the continuity of $F$ at $t_{0}$. Thus $F$ is continuous on $[a, b]$.

Now, we are ready to formulate the main result of this work.

Theorem 3.1. Let $(X,\|\cdot\|),(Y,\|\cdot\|)$ be normed spaces, $K$ be a convex cone in $X$ and $\varphi_{1}, \varphi_{2}$ be two convex $\varphi$-functions in $X$, strictly increasing, 
satisfying condition $\infty_{1}$ and such that there exist constants $c$ and $T_{0}$ with $\varphi_{2}(t) \leq \varphi_{1}(c t)$ for all $t \geq T_{0}$. If the Nemytskiu operator $N$ generated by a set-valued function $H:[a, b] \times K \rightarrow C C(Y)$ maps $R V_{\varphi_{1}}([a, b] ; K)$ into $R W_{\varphi_{2}}([a, b] ; C C(Y))$ and if it is globally Lipschitz, then the set-valued function $H$ satisfies the following conditions:

(a) For every $t \in[a, b]$ there exists $M(t) \in[0, \infty)$, such that

$$
D(H(t, x), H(t, y)) \leq M(t)\|x-y\|, \quad x, y \in K .
$$

(b) There are $A:[a, b] \rightarrow L(K ; C C(Y))$ and $B \in R W_{\varphi_{2}}([a, b] ; C C(Y))$ such that

$$
H(t, x)=A(t) x+B(t), \quad t \in[a, b], x \in K .
$$

Proof. (a) Since $N$ is globally Lipschitz, there exists a constant $M \in$ $[0, \infty)$ such that

$$
D_{\varphi_{2}}(N u, N v) \leq M\|u-v\|_{\varphi_{1}}, \quad u, v \in R V_{\varphi_{1}}([a, b] ; K) .
$$

Using the definition of $N$ and $D_{\varphi_{2}}$ we obtain

$$
\begin{gathered}
D(N u(a), N v(a)) \\
+\inf \left\{\varepsilon>0: \sup _{\pi} \sum_{i=1}^{n} \varphi_{2}\left(\frac{D\left(h_{t_{i}, t_{i-1}} N_{u, v}, h_{t_{i-1}, t_{i}} N_{u, v}\right)}{\varepsilon\left|t_{i}-t_{i-1}\right|}\right)\left|t_{i}-t_{i-1}\right| \leq 1\right\} \\
\leq M\|u-v\|_{\varphi_{1}}, \quad u, v \in R V_{\varphi_{1}}([a, b] ; K),
\end{gathered}
$$

where $h_{s, t} N_{u, v}:=(N u)(s)+(N v)(t)$. In particular,

$$
\inf \left\{\varepsilon>0: \varphi_{2}\left(\frac{D\left(d_{u, v}(H, t, \bar{t}), d_{u, v}(H, \bar{t}, t)\right)}{\varepsilon|\bar{t}-t|}\right)|\bar{t}-t| \leq 1\right\} \leq M\|u-v\|_{\varphi_{1}} \text {, }
$$

for all $u, v \in R V_{\varphi}([a, b] ; K)$ and $t, \bar{t} \in[a, b], t \neq \bar{t}$, where $d_{u, v}(H, s, t)=$ $H(s, u(s))+H(t, v(t))$. Since $\varphi_{1}$ and $\varphi_{2}$ satisfy

$$
\varphi_{i}\left(\varphi_{i}^{-1}\left(\frac{1}{|\bar{t}-t|}\right)\right)|\bar{t}-t|=1, \quad i=1,2,
$$

we obtain

$$
\begin{aligned}
\inf \left\{\varepsilon>0: \varphi_{2}\left(\frac{D\left(d_{u, v}(H, t, \bar{t}), d_{u, v}(H, \bar{t}, t)\right)}{\varepsilon|\bar{t}-t|}\right)|\bar{t}-t| \leq 1\right\} & \\
& =D\left(d_{u, v}(H, t, \bar{t}), d_{u, v}(H, \bar{t}, t)\right) .
\end{aligned}
$$

Therefore

$$
D\left(d_{u, v}(H, t, \bar{t}), d_{u, v}(H, \bar{t}, t)\right) \leq M\|u-v\|_{\varphi_{1}}|\bar{t}-t| \varphi_{2}^{-1}\left(\frac{1}{|\bar{t}-t|}\right) .
$$

Now, fix $t \in(a, b]$ and consider the function $\alpha:[a, b] \rightarrow[0,1]$ defined by 


$$
\alpha(s):= \begin{cases}\frac{s-a}{t-a}, & a \leq s \leq t \\ 1, & t \leq s \leq b .\end{cases}
$$

Then $\alpha \in R V_{\varphi_{1}}[a, b]$ and $V_{\varphi_{1}}(\alpha ;[a, b])=\varphi_{1}\left(\frac{1}{|t-a|}\right)|t-a|$.

Fix $x, y \in K$ and define $u, v:[a, b] \rightarrow K$ by

$$
u(s):=x, \quad v(s):=\alpha(s)(y-x)+x, \quad s \in[a, b] .
$$

Then $u, v \in R V_{\varphi_{1}}([a, b] ; K)$ and

$$
+\inf \left\{\varepsilon>0: \sup _{\pi} \sum_{i=1}^{n} \varphi_{1}\left(\frac{\left\|(u-v)\left(t_{i}\right)-(u-v)\left(t_{i-1}\right)\right\|}{\varepsilon\left|t_{i}-t_{i-1}\right|}\right)\left|t_{i}-t_{i-1}\right| \leq 1\right\} .
$$

From the definition of $u$ and $v$ we have

$$
\|u-v\|_{\varphi_{1}}=\inf \left\{\varepsilon>0: \varphi_{1}\left(\frac{\|x-y\|}{\varepsilon|t-a|}\right)|t-a| \leq 1\right\} .
$$

From (3.23) we get

$$
\inf \left\{\varepsilon>0: \varphi\left(\frac{\|x-y\|}{\varepsilon|t-a|}\right)|t-a| \leq 1\right\}=\frac{\|x-y\|}{|t-a| \varphi_{1}^{-1}\left(\frac{1}{|t-a|}\right)} .
$$

Hence,

$$
D\left(d_{u, v}(H, t, \bar{t}), d_{u, v}(H, \bar{t}, t)\right) \leq \frac{M|\bar{t}-t| \varphi_{2}^{-1}\left(\frac{1}{|\bar{t}-t|}\right)\|x-y\|}{|t-a| \varphi_{1}^{-1}\left(\frac{1}{|t-a|}\right)} .
$$

Hence, substituting in (3.24) the particular functions $u$ and $v$ defined above, and taking $\bar{t}=a$ in (3.30), we get

$$
D(H(t, x)+H(a, x), H(a, x)+H(t, y)) \leq M \frac{\varphi_{2}^{-1}\left(\frac{1}{|t-a|}\right)}{\varphi_{1}^{-1}\left(\frac{1}{|t-a|}\right)}\|x-y\|
$$

for all $t \in(a, b]$ and $x, y \in K$. By Lemma 2.1 and the above inequality,

$$
D(H(t, x), H(t, y)) \leq M \frac{\varphi_{2}^{-1}\left(\frac{1}{|t-a|}\right)}{\varphi_{1}^{-1}\left(\frac{1}{|t-a|}\right)}\|x-y\|
$$

for all $t \in(a, b]$ and $x, y \in K$.

Now, we have to consider the case $t=a$. Define $\beta:[a, b] \rightarrow[0,1]$ by

$$
\beta(s):=\frac{s-a}{b-a}, \quad s \in[a, b] .
$$

Then $\beta \in R V_{\varphi_{1}}[a, b]$ and $V_{\varphi_{1}}(\beta ;[a, b])=\varphi_{1}\left(\frac{1}{|b-a|}\right)|b-a|$.

Fix $x, y \in K$ and define $u, v:[a, b] \rightarrow K$ by

$$
u(s):=x, \quad v(s):=\beta(s)(x-y)+y, \quad s \in[a, b] .
$$


Then $u, v \in R V_{\varphi_{1}}([a, b] ; K)$ and

$$
\begin{aligned}
\|u-v\|_{\varphi_{1}} & =\|x-y\|+\inf \left\{\varepsilon>0: \varphi_{1}\left(\frac{\|x-y\|}{\varepsilon|b-a|}\right)|b-a| \leq 1\right\} \\
& =\|x-y\|+\frac{\|x-y\|}{\|b-a\| \varphi_{1}^{-1}\left(\frac{1}{|b-a|}\right)} \\
& =\|x-y\|\left(1+\frac{1}{|b-a| \varphi_{1}^{-1}\left(\frac{1}{|b-a|}\right)}\right) .
\end{aligned}
$$

Substituting $\bar{t}=a$ and $t=b$ in (3.24), we get

$$
D(H(b, x)+H(a, y), H(a, x)+H(b, x)) \leq M K\left(a, b, x, y, \varphi_{1}^{-1}, \varphi_{2}^{-1}\right)
$$

for all $x, y \in K$, where

$$
\begin{aligned}
& K\left(a, b, x, y, \varphi_{1}^{-1}, \varphi_{2}^{-1}\right) \\
& \quad=|b-a| \varphi_{2}^{-1}\left(\frac{1}{|b-a|}\right)\|x-y\|\left(1+\frac{1}{|b-a| \varphi_{1}^{-1}\left(\frac{1}{|b-a|}\right)}\right) .
\end{aligned}
$$

By Lemma 2.1 and the last inequality we get

$$
\begin{aligned}
& D(H(a, x), H(a, y)) \\
& \quad \leq M|b-a| \varphi_{2}^{-1}\left(\frac{1}{|b-a|}\right)\|x-y\|\left(1+\frac{1}{|b-a| \varphi_{1}^{-1}\left(\frac{1}{|b-a|}\right)}\right)
\end{aligned}
$$

for all $x, y \in K$.

Define $M:[a, b] \rightarrow \mathbb{R}$ by

$$
M(t):=\left\{\begin{array}{l}
M \frac{\varphi_{2}^{-1}\left(\frac{1}{|t-a|}\right)}{\varphi_{1}^{-1}\left(\frac{1}{|t-a|}\right)}, \quad t \in(a, b] \\
M|b-a| \varphi_{2}^{-1}\left(\frac{1}{|b-a|}\right)\left(1+\frac{1}{|b-a| \varphi_{1}^{-1}\left(\frac{1}{|b-a|}\right)}\right), \quad t=a .
\end{array}\right.
$$

Hence

(3.39) $D(H(t, x), H(t, y)) \leq M(t)\|x-y\|, \quad x, y \in X, t \in[a, b]$.

Consequently, for every $t \in[a, b]$ the function $H(t, \cdot): K \rightarrow C C(Y)$ is continuous.

(b) Fix $t, t_{0} \in[a, b]$ such that $t_{0}<t$. Since $N$ is globally Lipschitz, there exists a constant $M>0$ such that

$$
D\left(d_{u, v}\left(H, t, t_{0}\right), d_{u, v}\left(H, t_{0}, t\right)\right) \leq M\left|t_{0}-t\right| \varphi_{2}^{-1}\left(\frac{1}{\left|t_{0}-t\right|}\right)\|u-v\|_{\varphi_{1}},
$$


where $d_{u, v}(H, s, t)=H(s, u(s))+H(t, v(t))$. Define $\gamma:[a, b] \rightarrow[0,1]$ by

$$
\gamma(s)= \begin{cases}\frac{s-a}{t_{0}-a}, & a \leq s \leq t_{0} \\ \frac{t-s}{t-t_{0}}, & t_{0} \leq s \leq t \\ 0, & t \leq s \leq b\end{cases}
$$

Then $\gamma \in R V_{\varphi_{1}}[a, b]$. Fix $x, y \in K$ and define $u, v:[a, b] \rightarrow K$ by

$$
\begin{aligned}
& u(s):=\frac{\gamma(s)}{2} x+\left(1-\frac{\gamma(s)}{2}\right) y, \\
& v(s):=\left(\frac{1+\gamma(s)}{2}\right) x+\left(\frac{1-\gamma(s)}{2}\right) y, \quad s \in[a, b] .
\end{aligned}
$$

Then $u, v \in R V_{\varphi_{1}}([a, b] ; K)$ and

$$
\|u-v\|=\|u(a)-v(a)\|=\frac{\|x-y\|}{2} .
$$

Hence, substituting in (3.40) the particular functions $u, v$ defined in (3.41), we obtain

$$
\begin{aligned}
D\left(H\left(t_{0}, x\right)+H(t, y), H(\right. & \left.\left.t_{0}, \frac{x+y}{2}\right)+H\left(t, \frac{x+y}{2}\right)\right) \\
& \leq M\left|t-t_{0}\right| \varphi_{2}^{-1}\left(\frac{1}{\left|t-t_{0}\right|}\right) \frac{\|x-y\|}{2} .
\end{aligned}
$$

Since $N$ maps $R V_{\varphi_{1}}([a, b] ; K)$ into $R W_{\varphi_{2}}([a, b] ; C C(Y))$, it follows that $H(\cdot, z)$ is continuous for all $z \in K$. Hence, letting $t_{0} \uparrow t$ in (3.43), we obtain

$$
D\left(H(t, x)+H(t, y), H\left(t, \frac{x+y}{2}\right)+H\left(t, \frac{x+y}{2}\right)\right)=0
$$

for all $t \in[a, b]$ and $x, y \in K$. Thus

$$
H\left(t, \frac{x+y}{2}\right)+H\left(t, \frac{x+y}{2}\right)=H(t, x)+H(t, y) .
$$

Since the values of $H$ are convex, we have

$$
H\left(t, \frac{x+y}{2}\right)=\frac{1}{2}\{H(t, x)+H(t, y)\}
$$

for all $t \in[a, b]$ and $x, y \in K$. Thus for all $t \in[a, b]$, the set-valued function $H(t, \cdot): K \rightarrow C C(Y)$ satisfies the Jensen equation (2.14). Now by Lemma 2.2, there exist an additive set-valued function $A(t): K \rightarrow C C(Y)$ and a set $B(t) \in C C(Y)$ such that

$$
H(t, x)=A(t)(x)+B(t), \quad x \in K, t \in[a, b] .
$$


From (3.47) and (3.39) we deduce that for all $t \in[a, b]$ there exists $M(t) \in$ $[0, \infty)$ such that

$$
D(A(t) x, A(t) y) \leq M(t)\|x-y\|, \quad x, y \in K .
$$

Consequently, for every $t \in[a, b]$ the set-valued function $A(t): K \rightarrow C C(Y)$ is continuous, and $A(t) \in L(K ; C C(Y))$. Since $A(t)$ is additive and $0 \in K$, we have $A(t)=0$ for all $t \in[a, b]$ and $H(\cdot, 0)=B(\cdot)$.

The Nemytskiu operator $N$ maps $R V_{\varphi_{1}}([a, b] ; K)$ into $R W_{\varphi_{2}}([a, b] ; C C(Y))$. Therefore $H(\cdot, 0)=B(\cdot) \in R W_{\varphi_{2}}([a, b] ; K)$. Consequently, the set-valued function $H$ has to be of the form $H(t, x)=A(t) x+B(t)$ for all $t \in[a, b]$ and $x \in K$, where $A(t) \in L(K ; C C(Y))$ and $B \in R W_{\varphi_{2}}([a, b] ; C C(Y))$.

Theorem 3.2. Let $(X,\|\cdot\|),(Y,\|\cdot\|)$ be normed spaces, $K$ be a convex cone in $X$ and $\varphi_{1}, \varphi_{2}$ be two convex $\varphi$-functions in $X$, strictly increasing, satifying condition $\infty_{1}$ and $\lim _{t \rightarrow \infty} \varphi_{2}^{-1}\left(\varphi_{1}(t)\right) / t=\infty$. If the Nemytskiu operator $N$ generated by a set-valued function $H:[a, b] \times K \rightarrow C C(Y)$ maps $R V_{\varphi_{2}}([a, b] ; K)$ into $R W_{\varphi_{1}}([a, b] ; C C(Y))$ and is globally Lipschitz, then

$$
H(t, x)=H(t, 0) \quad \text { for } t \in[a, b], x \in K
$$

i.e. the Nemytskiu operator is constant.

Proof. Since $N$ is globally Lipschitz from $R V_{\varphi_{2}}([a, b] ; K)$ to $R W_{\varphi_{1}}([a, b]$; $C C(Y)$ ), there exists a constant $M$ such that

$$
D_{\varphi_{1}}(N u, N v) \leq M\|u-v\|_{\varphi_{2}}, \quad u, v \in R V_{\varphi_{2}}([a, b] ; K) .
$$

Fix $t, t_{0} \in[a, b]$ such that $t_{0}<t$. Using the definition of $N$ and $D_{\varphi_{1}}$ we obtain

$$
D\left(d_{u, v}\left(H, t, t_{0}\right), d_{u, v}\left(H, t_{0}, t\right)\right) \leq M\|u-v\|_{\varphi_{2}}\left|t-t_{0}\right| \varphi_{1}^{-1}\left(\frac{1}{\left|t-t_{0}\right|}\right)
$$

for $u, v \in R V_{\varphi_{2}}([a, b] ; K)$.

Define $\alpha:[a, b] \rightarrow[0,1]$ by

$$
\alpha(s):= \begin{cases}1, & a \leq s \leq t_{0} \\ -\frac{s-t}{t-t_{0}}, & t_{0} \leq s \leq t \\ 0, & t \leq s \leq b\end{cases}
$$

Then $\alpha \in R V_{\varphi_{2}}[a, b]$ and $V_{\varphi_{2}}(\alpha ;[a, b])=\left|t-t_{0}\right| \varphi_{2}^{-1}\left(\frac{1}{\left|t-t_{0}\right|}\right)$.

Fix $x \in K$ and define $u, v:[a, b] \rightarrow K$ by

$$
u(s):=x, \quad v(s):=\alpha(s) x, \quad s \in[a, b] .
$$

Then $u, v \in R V_{\varphi_{2}}([a, b] ; K)$ and 


$$
\begin{array}{r}
+\inf \left\{\varepsilon>0: \sup _{\pi} \sum_{i=1}^{n} \varphi_{2}\left(\frac{\|(u-v)\left(t_{i}\right)-(u-v)\left(t_{i-1} \|\right.}{\varepsilon\left|t_{i}-t_{i-1}\right|}\right)\left|t_{i}-t_{i-1}\right| \leq 1\right\} \\
\quad=\inf \left\{\varepsilon>0: \varphi_{2}\left(\frac{\|x\|}{\varepsilon\left|t-t_{0}\right|}\right)\left|t-t_{0}\right| \leq 1\right\}=\frac{\|x\|}{\left|t-t_{0}\right| \varphi_{2}^{-1}\left(\frac{1}{\left|t-t_{0}\right|}\right)} .
\end{array}
$$

Hence, substituting in (3.50) the particular functions $u, v$ defined in (3.51), we obtain

$$
\begin{aligned}
& D\left(H(t, x)+H\left(t_{0}, x\right), H\left(t_{0}, x\right)+H(t, 0)\right) \\
& \leq M\left|t-t_{0}\right| \frac{\varphi_{1}^{-1}\left(\frac{1}{\left|t-t_{0}\right|}\right)}{\left|t-t_{0}\right| \varphi_{2}^{-1}\left(\frac{1}{\left|t-t_{0}\right|}\right)}\|x\| .
\end{aligned}
$$

Then

$$
D\left(H(t, x)+H\left(t_{0}, x\right), H\left(t_{0}, x\right)+H(t, 0)\right) \leq M \frac{\varphi_{1}^{-1}\left(\frac{1}{\left|t-t_{0}\right|}\right)}{\varphi_{2}^{-1}\left(\frac{1}{\left|t-t_{0}\right|}\right)}\|x\| .
$$

By Lemma 2.1 and the above inequality, we get

$$
D(H(t, x), H(t, 0)) \leq M\|x\| \frac{\varphi_{1}^{-1}\left(\frac{1}{\left|t-t_{0}\right|}\right)}{\varphi_{2}^{-1}\left(\frac{1}{\left|t-t_{0}\right|}\right)} .
$$

Since $\lim _{t \rightarrow \infty} \varphi_{2}^{-1}\left(\varphi_{1}(t)\right) / t=\infty$, letting $t_{0} \uparrow t$ in the last inequality, we have

$$
D(H(t, x), H(t, 0))=0 .
$$

Thus for all $t \in[a, b]$ and for all $x \in K$, we get

$$
H(t, x)=H(t, 0) .
$$

Theorem 3.3. Let $(X,\|\cdot\|),(Y,\|\cdot\|)$ be normed spaces, $K$ be a convex cone in $X$ and $\varphi$ be a convex $\varphi$-function in $X$ satisfying condition $\infty_{1}$. If the Nemytski乞 operator $N$ generated by a set-valued function $H:[a, b] \times$ $K \rightarrow C C(Y)$ maps $R V_{\varphi}([a, b] ; K)$ into $B W([a, b] ; C C(Y))$ and is globally Lipschitz, then the left regularization $H^{*}:[a, b] \times K \rightarrow C C(Y)$ of $H$ defined by

$$
H^{*}(t, x)= \begin{cases}H^{-}(t, x), & t \in(a, b], x \in K, \\ \lim _{s \downarrow a} H(s, x), & t=a, x \in K,\end{cases}
$$

satisfies the following conditions:

(a) For every $t \in[a, b]$ there exists $M(t) \in[0, \infty)$ such that

$$
D_{1}\left(H^{*}(t, x), H^{*}(t, y)\right) \leq M(t)\|x-y\|, \quad x, y \in K .
$$


(b) There are functions $A:[a, b] \rightarrow L(K ; C C(Y))$ and $B \in B W([a, b]$; $C C(Y))$ such that

$$
H^{*}(t, x)=A(t) x+B(t), \quad t \in[a, b], x \in K .
$$

Proof. Fix $t \in[a, b]$ and consider $\alpha:[a, b] \rightarrow[0,1]$ defined by

$$
\alpha(s):= \begin{cases}1, & a \leq s \leq t \\ \frac{s-b}{t-b}, & t \leq s \leq b\end{cases}
$$

Then $\alpha \in R V_{\varphi}[a, b]$ and $V_{\varphi}(\alpha ;[a, b])=\varphi\left(\frac{1}{|t-b|}\right)|t-b|$.

Fix $x, y \in K$ and define $u, v:[a, b] \rightarrow K$ by

$$
u(s):=x, \quad v(s):=\alpha(s)(y-x)+x, \quad s \in[a, b] .
$$

Then $u, v \in R V_{\varphi}([a, b] ; K)$ and

$$
+\inf \left\{\varepsilon>0: \sup _{\pi} \sum_{i=1}^{n} \varphi\left(\frac{\left\|(u-v)\left(t_{i}\right)-(u-v)\left(t_{i-1}\right)\right\|}{\varepsilon\left|t_{i}-t_{i-1}\right|}\right)\left|t_{i}-t_{i-1}\right| \leq 1\right\} .
$$

From the definition of $u$ and $v$ we obtain

$$
\|u-v\|_{\varphi}=\|x-y\|+\left(1+\frac{1}{|b-t| \varphi^{-1}\left(\frac{1}{|b-t|}\right)}\right) .
$$

Since $N$ is globally Lipschitz, there exists a constant $M>0$ such that

$$
D(H(b, u(b))+H(t, v(t)), H(t, u(t))+H(b, v(b))) \leq M\|u-v\|_{\varphi}
$$

for $u, v \in R V_{\varphi}([a, b] ; K)$. Substituting $u, v$ defined by (3.61), we obtain

$$
D(H(b, x)+H(t, y), H(b, x)+H(t, x)) \leq M(t)\|x-y\|_{\varphi}\left(1+\frac{1}{|b-t| \varphi^{-1}\left(\frac{1}{|b-t|}\right)}\right)
$$

for all $t \in[a, b)$ and $x, y \in K$. By Lemma 2.1 we get

$$
D(H(t, x), H(t, y)) \leq M(t)\|x-y\|_{\varphi}\left(1+\frac{1}{|b-t| \varphi^{-1}\left(\frac{1}{|b-t|}\right)}\right)
$$

for all $t \in[a, b)$ and $x, y \in K$.

For $t=b$, by a similar reasoning, we show that there exists a constant $M(b)>0$ such that

$$
D(H(t, x), H(t, y)) \leq M(b)\|x-y\|_{\varphi} \quad \text { for } x, y \in K .
$$

Define the function $M:[a, b] \rightarrow \mathbb{R}$ by

$$
M(t):= \begin{cases}M\left(1+\frac{1}{|b-t| \varphi^{-1}\left(\frac{1}{|b-t|}\right)}\right), & a \leq t<b, \\ M(b), & t=b .\end{cases}
$$




\section{Hence}

$$
D(H(t, x), H(t, y)) \leq M(t)\|x-y\|_{\varphi}, \quad t \in[a, b], x, y \in K .
$$

Passing to the limit in (3.64) and by the inequality (3.66) and the definition of $H^{*}$ we conclude that for all $t \in[a, b]$ there exists $M(t) \in[0, \infty)$ such that

$$
D\left(H^{*}(t, x), H^{*}(t, y)\right) \leq M(t)\|x-y\| \quad \text { for } x, y \in K .
$$

Now we shall prove that

$$
H^{*}(t, x)=A(t) x+B(t), \quad t \in[a, b], x \in K,
$$

where $A(t)$ is a linear continuous set-valued function, and $B \in B W([a, b]$; $C C(Y))$.

Fix $t, t_{0} \in[a, b]$ with $t_{0}<t$ and $n \in \mathbb{N}$. Define the partition $\pi_{n}$ of the interval $\left[t_{0}, t\right]$ by

$$
\pi_{n}: a<t_{0}<t_{1}<\cdots<t_{2 n-1}<t_{2 n}=t, \quad \text { where } \quad t_{i}-t_{i-1}=\frac{t-t_{0}}{2 n},
$$
$i=1, \ldots, 2 n$.

Since $N$ is globally Lipschitz from $R V_{\varphi}([a, b] ; K)$ to $B W([a, b] ; C C(Y))$, there exists a constant $M>0$ such that

$$
\left.\sum_{i=1}^{n} D\left(d_{u, v}\left(H, t_{2 i}, t_{2 i-1}\right), d_{u, v}\left(H, t_{2 i-1}, t_{2 i}\right)\right)\right) \leq M\|u-v\|_{\varphi}
$$

where $u, v \in R V_{\varphi}([a, b] ; K)$ and $d_{u, v}(H, s, t)=H(s, u(s))+H(t, v(t))$.

Define $\alpha:[a, b] \rightarrow[0,1]$ in the following way:

$$
\alpha(s):= \begin{cases}0, & a \leq s \leq t_{i-1}, \\ \frac{s-t_{i-1}}{t_{i}-t_{i-1}}, & t_{i-1} \leq s \leq t_{i}, i=1,3, \ldots, 2 n-1, \\ -\frac{s-t_{i}}{t_{i}-t_{i-1}}, & t_{i-1} \leq s \leq t_{i}, i=2,4, \ldots, 2 n, \\ 0, & t_{i} \leq s \leq b .\end{cases}
$$

Then $\alpha \in R V_{\varphi}([a, b] ; K)$ and $V_{\varphi}(\alpha ;[a, b])=\left|t-t_{0}\right| \varphi\left(\frac{2 n}{\left|t-t_{0}\right|}\right)$.

Fix $x, y \in K$ and define $u, v:[a, b] \rightarrow K$ by

$$
\begin{array}{ll}
u(s):=\frac{\alpha(s)}{2} x+\left(1-\frac{\alpha(s)}{2}\right) y, & s \in[a, b] . \\
v(s):=\frac{1+\alpha(s)}{2} x+\frac{1-\alpha(s)}{2} y, \quad s \in[a, b] .
\end{array}
$$

Then $u, v \in R V_{\varphi}([a, b] ; K)$ and $\|u-v\|_{\varphi}=\|x-y\| / 2$. Hence, substituting in (3.67) the particular functions $u, v$ defined in (3.69), we obtain 


$$
\begin{array}{r}
\sum_{i=1}^{n} D\left(H\left(t_{2 i}, y\right)+H\left(t_{2 i-1}, x\right), H\left(t_{2 i}, \frac{x+y}{2}\right)+H\left(t_{2 i-1}, \frac{x+y}{2}\right)\right) \\
\leq M \frac{\|x-y\|}{2}
\end{array}
$$

for all $x, y \in K$. Since $N$ maps $R V_{\varphi}([a, b] ; K)$ into $B W([a, b] ; C C(Y))$, it follows that $H(\cdot, z) \in B W([a, b] ; C C(Y))$ for all $z \in K$. Hence, letting $t_{0} \uparrow t$ in (3.70), we get

$$
\begin{aligned}
& D\left(H^{*}(t, y)+H^{*}(t, x), H^{*}\left(t, \frac{x+y}{2}\right)+H^{*}\left(t, \frac{x+y}{2}\right)\right) \\
& \leq M \frac{\|x-y\|}{2 n}
\end{aligned}
$$

for all $x, y \in K$ and $n \in \mathbb{N}$. Passing to the limit as $n \rightarrow \infty$ we get

$$
H^{*}\left(t, \frac{x+y}{2}\right)+H^{*}\left(t, \frac{x+y}{2}\right)=H^{*}(t, y)+H^{*}(t, x)
$$

for all $x, y \in K$ and $t \in[a, b]$.

Since $H^{*}(t, x)$ is a convex set, we have

$$
H^{*}\left(t, \frac{x+y}{2}\right)=\frac{1}{2}\left(H^{*}(t, y)+H^{*}(t, x)\right)
$$

for all $x, y \in K$ and $t \in[a, b]$.

Thus for every $t \in[a, b]$ the set-valued function $H^{*}(t, \cdot): K \rightarrow C C(Y)$ satisfies the Jensen equation (2.14). By Lemma 2.2, for all $t \in[a, b]$ there exist an additive set-valued function $A(t): K \rightarrow C C(Y)$ and a set $B(t) \in$ $C C(Y)$ such that

$$
H^{*}(t, x)=A(t) x+B(t) \quad \text { for } t \in[a, b] \text { and } x \in K .
$$

Acknowledgements. We would like to express our thanks to the referee for his valuable suggestions improving our paper.

\section{References}

[1] H. Herda, Modular spaces of generalized variation, Studia Math. 30 (1968), 21-42.

[2] L. Maligranda and W. Orlicz, On some properties of functions of generalized variation, Monatsh. Math. 104 (1987), 53-65.

[3] J. Matkowski, Functional equations and Nemytskiu operators, Funkcial. Ekvac. 25 (1982), 127-132.

[4] J. Matkowski and J. Miś, On a characterization of Lipschitzian operators of substitution in the space BV [a,b], Math. Nachr. 117 (1984), 155-159.

[5] N. Merentes and K. Nikodem, On Nemytskii operator and set-valued functions of bounded p-variation, Rad. Mat. 8 (1996), 139-145.

[6] N. Merentes, K. Nikodem and S. Rivas, Continuity of the superposition of set-valued functions, J. Appl. Anal. 3 (1997), 43-48. 
[7] N. Merentes and S. Rivas, On Nemytskiu operator in the space of set-valued functions of bounded p-variation in the sense of Riesz, Publ. Math. Debrecen 47 (1995), 15-27.

[8] K. Nikodem, K-convex and K-concave set-valued functions, Politech. Łódz., Zeszyty Nauk. 559, Rozprawy Naukowe Z 114, Łódź, 1989.

[9] H. Radström, An embedding theorem for space of convex sets, Proc. Amer. Math. Soc. 3 (1952), 165-169.

[10] F. Riesz, Untersuchugen über Systeme integrierbarer Funktionen, Math. Ann. 69 (1910), 449-497.

[11] A. Smajdor and W. Smajdor, Jensen equation and Nemytskij operator for set-valued functions, Rad. Mat. 5 (1989), 311-319.

[12] G. Zawadzka, On Lipschitzian operators of substitution in the space of set-valued functions of bounded variation, ibid. 6 (1990), 179-193.

Nelson Merentes

Departamento de Matemática

Universidad Central de Venezuela

Caracas 1020A, Venezuela

E-mail: merentes@euler.ciens.ucv.ve
Jose Luis Sánchez Hernández

School of Mathematics

Georgia Tech

Atlanta, GA 30332-0160, U.S.A. E-mail: sanchez@math.gatech.edu 